\title{
Heart Rate Variability during Plateau Waves of Intracranial Pressure: a pilot descriptive study
}

\author{
A. Luís, A.S. Santos, C. Dias, R. Almeida, A.P. Rocha, Member, IEEE
}

\section{INTRODUCTION}

In 1950 Janny first described sudden rises in intracranial pressure (ICP), but the term plateau waves or A waves was proposed by Lundberg in 1960 [1].

Plateau waves (PW) are defined by a sudden substantial elevation in ICP, up to $40-100 \mathrm{mmHg}$ that lasts more than 5 minutes [2] and terminates either spontaneously or in response to active treatment, [3] while arterial blood pressure remains stable or varies modestly.

The classic accepted mechanism of a plateau wave is a vasogenic positive feedback loop triggered by active vasodilatation, that increases cerebral blood volume (CBV), leading to elevation of ICP, decrease of cerebral perfusion pressure (CPP), further vasodilation until maximum vasodilation is reached. A vasoconstrictive stimulus (either therapeutic or spontaneous) stops this mechanism, reversing this loop and restoring ICP [2]. These waves are not usually associated with worse outcome, unless they last longer than 30 min [4]. Nevertheless, these acute elevations of ICP may lead to dramatic decreases of CPP, which can, therefore

*Research partially supported by CMUP (UID/MAT/00144/2013), funded by FCT (Portugal) with national (MEC) and European structural funds through FEDER, under the partnership agreement PT2020. Study partially supported by project TEC2013-42140-R from MICINN, Spain, CIBER, a center of the Instituto de Salud Carlos III with assistance from the European Regional Development and by Grupo Consolidado. BSICoS from DGA (Aragón, Spain) and European Social Fund (EU).

A. Luís and A. S. Santos are with the Centro Hospitalar de Lisboa Ocidental (e-mail: analuis.nc@gmail.com, alexandrasantos@campus.ul.pt). A. S. Santos also with Faculty of Medicine of Lisbon, Lisbon, Portugal. C. Dias is with Centro Hospitalar São João and Faculty of Medicine, University of Porto, Porto, Portugal (e-mail: mceleste.dias@gmail.com). R. Almeida and A. P. Rocha are with Departamento de Matematica, Faculdade de Ciências, Universidade do Porto (FCUP), Centro de Matematica da Universidade do Porto (CMUP), Rua do Campo Alegre, 687, 4169-007 Porto, Portugal (e-mail: rbalmeid@fc.up.pt, aprocha@fc.up.pt). R. Almeida (corresponding author) is also with BSICoS Group. Aragon Institute for Engineering Research (I3A). IIS Aragon, Spain. (phone: 00351- 220402 281; fax: 00351-220 402 108). induce reduction of brain blood flow and tissue oxygenation, contributing to secondary brain lesion [5].

Plateau waves are related with cerebrovascular reactivity [2] and exhausted cerebrospinal compensatory reserve, thus are common cerebrovascular phenomena in several neurological and neurosurgical conditions, including: traumatic brain injury $[2,3,4]$, subarachnoid hemorrhage [6,7], intracerebral hemorrhage [7], brain tumors [8], craniosynostosis [9], idiopathic intracranial pressure [7,10], leptomeningeal carcinomatosis and encephalitis [11].

The autonomic nervous system plays a role in cerebrovascular reactivity and triggers systemic vascular changes. Therefore a quantitative analysis of autonomic function (such as heart rate variability - HRV) may provide more information about PW pathophysiology [5].

HRV (beat-to-beat variations in heart rate) may be studied using either time domain analysis or spectral analysis (Task Force of the European Society of Cardiology and the North American Society of Pacing and Electrophysiology, 1996) [12]. It has been showed that the $\mathrm{HF}$ component is related to the respiratory sinus arrhythmia and vagal activity of the heart while the LF component reflects the global activity of the autonomic nervous system $[13,14]$. HRV reduction was observed in brain injury patients, both in adult and pediatric patients [15], was associated with prognosis $[15,16]$ and can be used to predict impaired cerebral autoregulation [17]. As the PW are probably a reflex of autonomic dysfunction, the authors hypothesized that there is a relationship between ICP plateau waves and HRV.

\section{MATERIAL AND MethodS}

\section{A. Experimental Data}

1) Patients: retrospective study of 7 adult traumatic brain injury patients (TBI), that developed $\mathrm{PW}$, admitted to the Neurocritical Care Unit (NCCU) at Hospital São João, Porto. Exclusion criteria were pregnancy and age $<18$ years old. Patients were sedated with propofol and/or midazolam and fentanyl and normoventilated. ICP and CPP management was achieved according to the NCCU protocol already published [18] approved by the hospital Ethics Committee. The patients were continuously monitored for ECG at $250 \mathrm{~Hz}$, heart rate (HR), arterial blood pressure (ABP), ICP, CPP and End-tidal $\mathrm{CO} 2\left(\mathrm{ETCO}_{2}\right)$ for the first 10 days. The intraparenchymal ICP probe was inserted in the frontal area of the worst side of the brain lesion. We used $\mathrm{ICM}+{ }^{\circledR}$ software to collect primary data and calculate secondary variables related to compensatory reserve and cerebrovascular reactivity. $19.1 \mathrm{~mm}$ 


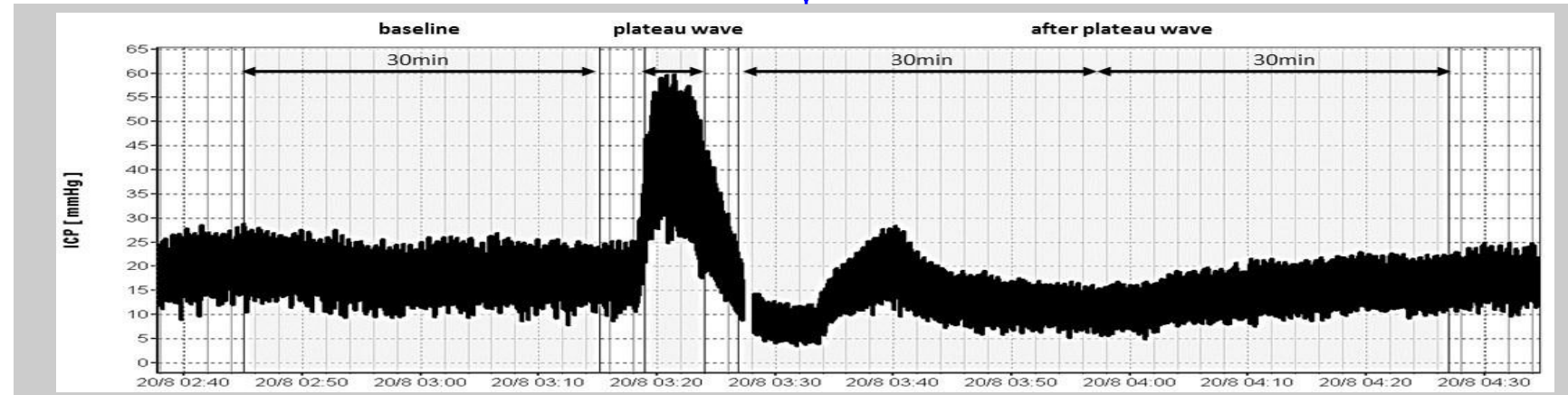

Figure 1 - Example of plateau wave (PW) of intracranial pressure (ICP) and analysis periods. Baseline - 30 min time interval before PW until ICP elevation $<20 \mathrm{mmHg}$. Plateau wave - time interval in which ICP $\geq 40 \mathrm{mmHg}$. After PW- $60 \mathrm{~min}$ time interval after PW, starting at ICP decrease $<20 \mathrm{mmHg}$.

2) Plateau Waves: primary clinical analysis of raw data included calculation of ten seconds averages of physiological variables HR (heart rate), ABP (arterial blood pressure), ICP, CPP and AMP (amplitude of ICP). We selected the first $\mathrm{PW}$ of all patients and divided it into three different periods of analysis, (Figure 1): A. baseline - 30 minutes period starting before PW and until ICP elevation $<20 \mathrm{mmHg}$. B. plateau wave - time interval in which ICP $\geq 40 \mathrm{mmHg}$. C. after PW -60 minutes time interval after plateau wave, starting at ICP $<20 \mathrm{mmHg}$. The entire interval was subdivided in nineteen segments of time for the analysis: b1-b6 are 5-minute intervals before the event (baseline), plw is the total time interval during the plateau wave and a1-a12 are 5-minute intervals after the event (after plw). For patient AS only a1-a10 were considered (50 minutes), as the remaining ECG signal was corrupted.

\section{B. Preprocessing and Data Analysis}

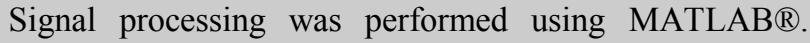
Demographic statistical analysis was performed using SPSS 23. Data were expressed as median values and range (min; max). Non-parametric Friedman tests were used to investigate statistical relationships between the studied variables over baseline, plw and after plw. Wilcoxon signedrank tests with Bonferroni correction were applied for the subsequent pairwise comparisons. Tests were considered statistically significant for $\mathrm{p}$ values $<0.05$.

Automatic delineation of the ECG signal was performed using a wavelet based approach described in [19] to efficiently extract RR series with no filtering or correction from the equipment. A local correction of the QRS mark was performed by taking the maximum amplitude sample in a centred 10 samples neighbourhood and using the integral pulse frequency modulation model [20], after which all beats are considered to be normal (NN). The RR series obtained as time differences of the corrected marks are then detrended using the approach proposed by Tarvainen [21]. Series were assumed to be sampled at the local mean heart rate, as this has been shown acceptable for spectral analysis for frequencies far from Nyquist frequency [20]

HRV analysis was performed according to the guidelines [12] for each of the twenty segments per patient available. The following time domain measures were obtained: RR intervals standard deviation (SDNN), standard deviation of differences between adjacent RR intervals (SDSD) and the square root of the mean on the sum of the squares of the differences between adjacent RR intervals (RMSSD).

For frequency domain analysis, standard fixed bands were considered [12] as low frequency (LF: 0.04-0.15 Hz), high frequency $(0.15-1.4 \mathrm{~Hz})$ bands and all frequencies in spectra (TP). Both non-parametric and parametric (ARautoregressive based) approaches were used. Welch method in 64 points windows, with $50 \%$ overlap and 512 points for fast Fourier transform estimation was used. Non parametric measures were taken as the total power (TPnp) and the power in each standard band (LFnp, HFnp), measured as the area under the spectra. AR identification was performed using forward-backward approach [22]. Only valid models with uncorrelated residual according to a $5 \%$ of significance Ljung-Box Q-test were admitted and best order selected from 6 to 20 according Akaike's Information criterion. If no admissible model was found, parametric analysis was not performed for that segment. Parametric HRV measures were obtained by assigning each pole contribution to the spectral band in which the pole is located (TPp, LFp, HFp) [23], using spectral AR decomposition.

\section{RESULTS}

\section{A. Demographic Data}

The study population included 7 adult patients with TBI: MT, AM, AR, AS, ACL, RMO and MCC (6 male; mean age 56 years; range, min-max: 20-82 years). The mean Glasgow coma score at admission was 7 (3-10) and the mean length of stay in NCCU was 27 days (12-37), with mean Glasgow outcome score at discharge 3 (1-5).

\section{B. Plateau Waves}

Physiological variables related to $\mathrm{PW}$ events are summarized in Table 1. The median duration of ICP waves was 5:07 $\mathrm{min}$ (02:07-13:07). The median amplitude of ICP (AMP) varied in accordance with the variation of ICP. Individual mean $\mathrm{ABP}$ remained almost constant at baseline, during $\mathrm{plw}$ and after plw $(p=0.5)$. Significant differences were found between baseline, plw and after plw for the remaining 3 physiological variables. Compared to baseline, CPP decreased and ICP and AMP increased during plw; compared to plw, CPP increased and ICP and AMP decreased after plw; $p=0.018$, Wilcoxon signed-rank tests with Bonferroni correction. 


\section{HRV Analysis}

As the clinical and time criteria were used to define the segments of analysis, a variable number of beats was found. The 5-min HR ranged from 41 to $78 \mathrm{bpm}$ at the baseline and 52 to $152 \mathrm{bpm}$ at after plw. The mean heart rate during PW ranged from 43-138 bpm and was higher than in b1 to b3 for all cases, but only for 2 of them increased by $10 \mathrm{bpm}$. Results from HRV analysis are presented in Figures 2 and 3.

With respect to time based HRV an increased variability was found during the latter part of the baseline and plw. The SDNN and SDSD in each segment and all patients are presented in Figure 2 a) and b). Behavior of RMSSD was found to be similar. Medians across patients, which is robust to outliers, were used to represent the global behavior (Figure 2c)).

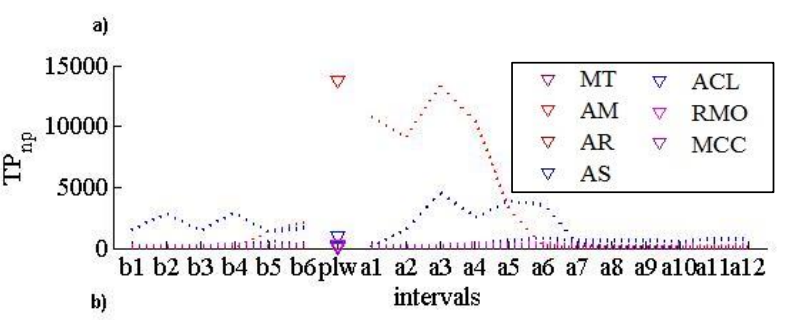

$\longleftrightarrow \underset{\substack{54 \mathrm{pt} \\ 0.75 \mathrm{in} \\ 19.1 \mathrm{~mm}}}{\longrightarrow}$
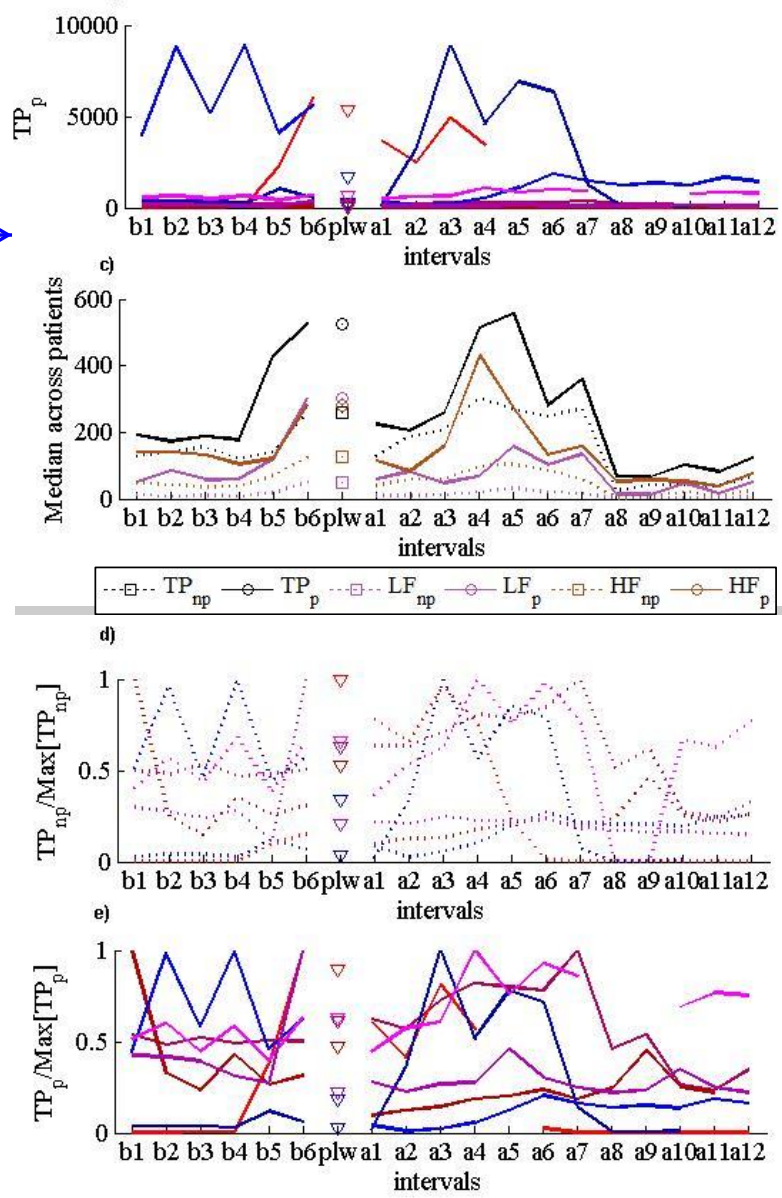

Figure 2 - a) RR intervals standard deviation (SDNN), b) standard deviation of differences between adjacent RR intervals (SDSD), c) median of SDNN and SDSD across patients, d) relative SDNN and e) relative SDSD, for each patient and interval.
As the variability of the measures across patients and segments is very high, SDNN and SDSD relative values (normalized by its maximum) are also presented in Figure 2 d) and e), for comparison purposes. All 7 patients present a rise of SDNN and SDSD at b5 or b6, preceding plw.

\begin{tabular}{lcccc}
$(\mathrm{mmHg})$ & baseline & plateau wave & after plw & $\boldsymbol{p}$ \\
\hline ABP & 104.2 & 108.1 & 98.9 & \\
& $(85.9 ; 115.4)$ & $(85.9 ; 123.5)$ & $(88.9 ; 118.0)$ & 0.5 \\
CPP & 83.7 & 68.8 & 80.1 & \\
& $(77.3 ; 101.9)$ & $(45.9 ; 77.6)$ & $(79.3 ; 100.2)$ & 0.005 \\
ICP & 18.1 & 41.4 & 17.5 & \\
& $(8.2 ; 23.7)$ & $(37.5 ; 45.9)$ & $(4.5 ; 21.4)$ & 0.02 \\
AMP & 2.7 & 10.2 & 2.9 & \\
& $(2.1 ; 12.3)$ & $(3.7 ; 13.4)$ & $(1.9 ; 10.0)$ & 0.005 \\
\hline
\end{tabular}

Table 1 - Median and range (min; max) across patients of mean arteria blood pressure $(\mathrm{ABP})$, cerebral perfusion pressure (CPP), intracranial pressure (ICP) and amplitude of ICP (AMP). Friedman tests $p$ values.
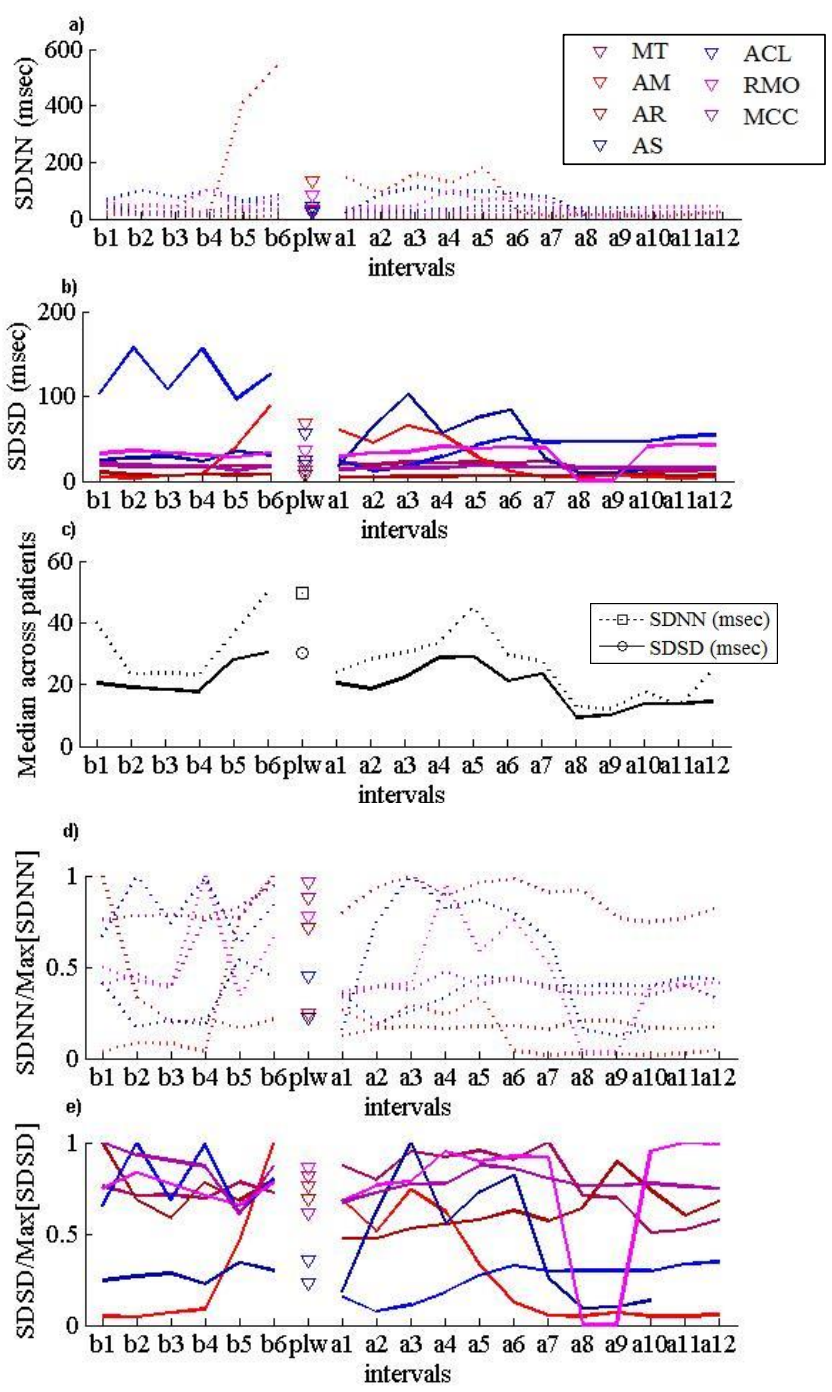

Figure 3 - a) Non parametric spectral power (TPnp), b) parametric spectral power (TPp), c) median across patients, d) relative TPnp and e) relative $\mathrm{TPp}$, for each frequency band and interval. 
The HRV spectral measures obtained using non parametric and parametric methods are not interchangeable and cannot be compared directly [22]. The values of TPnp and TPp for all patients and each interval are presented in Figure 3 a) and b), respectively. The general behavior expressed in the medians across patients for each frequency band is presented in Figure 3c). As for time based measures, relative TP values are reported in Figure 3 d) and e). All 7 patients present a TPnp rise at $\mathrm{b} 5$ or $\mathrm{b} 6$, in accordance with HRV time based measures. In TPp this increase in seen in 6 patients, with MT patient remaining in same level. Increased HRV is seen in the latter part of the baseline (b5 and b6) and plw, followed by a decrease after the event and a new increase during the recovery. The increase preceding plw is more marked in the parametric analysis (Figure $3 \mathrm{c}$ )), which can indicate that AR modeling is more powerful for the study of this data rather than Welch (non parametric). As a matter of fact, applying AR spectral decomposition assigns the contribution of a pole totally to the same band, even if the tails cross its boundary. This overcomes in a certain way the limitation of fixed frequency bands LF and HF [24]. This issue can be of utmost importance in the case of intrisinc dynamic situations such as is the case of PW management.

\section{CONCLUSION}

Plateau waves of ICP are important cerebrovascular phenomena in TBI patients and seems to be expressed in HRV. In fact, the general behavior of the median HRV spectral measures across patients is concordant with an increased HRV in the latter part of the baseline and plateau $\rightarrow$ wave, followed by a decrease after the event and a new increase during the recovery. In LF and HF bands the same increase is more marked in the parametric analysis. Changes in the autonomic nervous system measured by HRV might trigger systemic vascular changes related to PW generation. Further studies are warranted to develop a bedside monitoring tool based on HRV to help clinicians to better identify the plateau waves and allow earlier management.

\section{REFERENCES}

[1] Lundberg N. "Continuous recording and control of ventricular fluid pressure in neurosurgical patients." Acta Psychiatr Neurol Scand. (1960) 149:158-67.

[2] Rosner MJ, Becker DP. "Origin and evolution of plateau waves. Experimental observations and a theoretical model." J Neurosurg. (1984) 60:312-24

[3] Varsos GV, de Riva N, Smielewski P, Pickard JD, Brady KM, Reinhard M, Avolio A, Czosnyka M. "Critical closing pressure during intracranial pressure plateau waves." Neurocrit Care. (2013) 18(3):341-8.

[4] Castellani G, Zweifel C, Kim DJ, et al. "Plateau waves in head injured patients requiring neurocritical care." Neurocrit Care. (2009) 11:143-50.

[5] Shahsavari S, McKelvey T, Ritzén CE, Rydenhag B. "Plateau waves and baroreflex sensitivity in patients with head injury: a case study." Conf Proc IEEE Eng Med Biol Soc. (2011) 37925.

[6] Hayashi M, Kobayashi H, Kawano H, Yamamoto S, Maeda T. "Cerebral blood flow and ICP patterns in patients with communicating hydrocephalus after aneurysm rupture." J Neurosurg. (1984) 61:30-6.

[7] Hayashi M, Handa Y, Kobayashi H, Kawano H, Ishii H, Hirose S. "Plateau-wave phenomenon (I). Correlation between the appearance of plateau waves and CSF circulation in patients with intracranial hypertension." Brain. (1991) 114 :2681-91.

[8] Matsuda M, Yoneda S, Handa H, Gotoh H. "Cerebral hemodynamic changes during plateau waves in brain-tumor patients." J Neurosurg. (1979) 50:483-8.

[9] Renier D, Sainte-Rose C, Marchac D, Hirsch JF. "Intracranial pressure in craniostenosis." J Neurosurg. (1982) 57:370-7.

[10] Stevens SA, Stimpson J, Lakin WD, Thakore NJ, Penar PL. “A model for idiopathic intracranial hypertension and associated pathological ICP wave-forms." IEEE Trans Bio-Med Eng. (2008) 55:388-98.

[11] Hansen K, Gjerris F, Sorensen PS. "Absence of hydrocephalus in spite of impaired cerebrospinal fluid absorption and severe intracranial hypertension." Acta Neurochir. (1987) 86:93-7.

[12] "Heart rate variability: standards of measurement, physiological interpretation and clinical use. Task Force of the European Society of Cardiology and the North American Society of Pacing and Electrophysiology." Circulation (1996) $1 ; 93(5): 1043-65$.

[13] Berger RD, Saul JP, Cohen RJ “ Transfer function analysis of autonomic regulation. I. Canine atrial rate response" Am J Physiol (1989) 256: H142-H52

[14] Chain-Fa Su, Terry B. Kuo, Jon-Son Kuo, Hsien-Yong Lai, Hsing I. Chen "Sympathetic and parasympathetic activities evaluated by heart-rate variability in head injury of various severities" Clinical Neurophysiology (2005) 116: 1273-79

[15] Brahm Goldstein, Daniel Toweill, Susanna Lai, Karen Sonnenthal, Bren Kimberly "Uncoupling of the autonomic and cardiovascular systems in acute brain injury" Am. J. Physiol. (1998) 275: R1287-R1292

[16] Ali Reza Vakilian, Farhad Iranmanesh, Ali Esmaeili Nadimi, Jafar Ahmadi Kahnali "Heart Rate Variability and QT Dispersion Study in Brain Death Patients and Comatose Patients with Normal Brainstem Function" Journal of the College of Physicians and Surgeons Pakistan (2011) 21 (3): 130-133

[17] Andrea Lavinio, Bogdan Ene-Iordache, Ilaria Nodari, Alan Girardini, Elena Cagnazzi, Frank Rasulo, Piotr Smielewski, Marek Czosnyka, Nicola Latronico "Cerebrovascular reactivity and autonomic drive following traumatic brain injury" Acta Neurochir Suppl (2008) 102: 3-7

[18] Celeste Dias, Maria João Silva, Eduarda Pereira, Elisabete Monteiro, Isabel Maia, Silvina Barbosa, Sofia Silva, Teresa Honrado, António Cerejo, Marcel J. H. Aries, Peter Smielewski, José-Artur Paiva, Marek Czosnyka. Optimal Cerebral Perfusion Pressure Management at Bedside: A SingleCenter Pilot Study. Neurocrit Care. (2015) Jan 8. [Epub ahead of print].

[19] Martinez JP, Almeida R, Olmos S, Rocha AP, Laguna P. "A wavelet-based ECG delineator: evaluation onn standard databases" IEEE TBE (2004) 51 (4):570-81.

[20] Mateo J, Laguna P. "Improved heart rate variability signal analysis from the beat occurrence times according to the IPFM model" IEEE TBE (2000) 47(8):985-96.

[21] Tarvainen MP, Ranta-aho, PO, Karjalainen, PA "An advanced detrending method with application to HRV analysis" IEEE TBE (2002) 49(2): 172 - 175.

[22] Pichon, A, Roulaud TM, Antoine-Jonville S, Bisschop C, Denjean A Spectral analysis of heart rate variability: interchangeability between autoregressive analysis and fast Fourier transform Journal of Electrocardiology (2006) 3931 3.

[23] R. Almeida, S. Gouveia, A. P. Rocha, E. Pueyo, J. P. Martínez, P. Laguna (2006). QT variability and HRV interactions in ECG: Quantification and Reliability. IEEE Trans. on Biomedical Engineering, 53, n.7, pp. 1317-1329.

[24] Costa O, Lago P, Rocha, AP;, Freitas J, Puig J; Carvalho MJ, de Freitas AF "The spectral analysis of heart rate variability. A comparative study between nonparametric and parametric spectral analysis in short series", Revista Portuguesa de Cardiologia (1995) 14: 621-6. 